\title{
The (im)possibility of collective risk measurement: Arrovian aggregation of variational preferences
}

\author{
Frederik S. Herzberg
}

Received: 9 February 2013 / Accepted: 12 March 2013 / Published online: 11 April 2013

(C) SAET 2013

\begin{abstract}
This paper studies collective decision making when individual preferences can be represented by convex risk measures. It addresses the question whether there exist non-dictatorial aggregation functions of convex risk measures satisfying the following Arrow-type rationality axioms: a weak form of universality, systematicity (a strong variant of independence), and the Pareto principle. Herein, convex risk measures are identified with variational preferences on account of the Maccheroni et al. (Econometrica 74(6):1447-1498, 2006) axiomatisation of variational preference relations and Föllmer's and Schied's (Finance Stoch 6(4):429-447, 2002; Stochastic Finance. An Introduction in Discrete Time. de Gruyter Studies in Mathematics, vol. 27, 2nd edn. de Gruyter, Berlin, 2004) representation theorem for concave monetary utility functionals. The cases of both finite and infinite electorates are considered.
\end{abstract}

\footnotetext{
This work has been partially supported by a German Research Foundation (DFG) grant, while the author visited the Mathematics Department of Princeton University and by a Visiting Fellowship of the Munich Center for Mathematical Philosophy (Ludwig Maximilian University of Munich). Talks based on earlier versions of this paper have been presented, among other audiences, to the 10th Society for the Advancement of Economic Theory (SAET) Conference on Current Trends in Economics (Singapore, August 2010), the conference Stochastics and Real-World Models (Bielefeld, July 2011) and the 2011 NSF/NBER/CEME Conference in Mathematical Economics and General Equilibrium Theory (Iowa City, October 2011). I would like to thank Professors Daniel Eckert, Edward Nelson, Konrad Podczeck, Frank Riedel and not least Nicholas Yannelis for helpful discussions and comments.
}

F. S. Herzberg $(\varangle)$

Institut für Mathematische Wirtschaftsforschung, Universität Bielefeld, Universitätsstraße 25, 33615 Bielefeld, Germany

e-mail: fherzberg@uni-bielefeld.de

\section{F. S. Herzberg}

Munich Center for Mathematical Philosophy, Ludwig-Maximilians-Universität München,

Ludwigstraße 31, 80539 Munich, Germany

e-mail: frederik.herzberg@1rz.uni-muenchen.de 
For finite electorates, we prove a variational analogue of Arrow's impossibility theorem. For infinite electorates, the possibility of rational aggregation depends on an equicontinuity condition for the variational preference profiles; we prove a variational analogue of Fishburn's possibility theorem and point to potential analogues of Campbell's impossibility theorem. The proof methodology is based on a model-theoretic approach to aggregation theory inspired by Lauwers and Van Liedekerke (J Math Econ 24(3):217-237, 1995). Diverse applications of these results are conceivable, in particular (a) the constitutional design of panels of risk managers and (b) microfoundations for (macroeconomic) multiplier preferences.

Keywords Arrow-type preference aggregation - Abstract aggregation theory Variational preferences · Convex risk measures · Ultraproduct

JEL Classification $\quad$ D71 $\cdot$ D81 $\cdot$ G11

\section{Introduction}

Consider a committee that has to compare several risky positions on a financial market. One obvious example from the recent past would be a panel of various supervisory authorities and public financial institutions, assessing various government bonds by means of several continuous convex risk measures. We ask the question whether it is possible to find a mechanism for merging, in a rational and systematic manner, the resulting differing risk assessments into a single risk assessment, one financial position at a time. It will turn out that for finite electorates, this is not the case; hence, a panel as in the above example should agree on a single risk measure from the outset. In the case of infinite electorates, however, we shall see that rational and systematic aggregation rules for preferences representing convex risk measures do exist; this observation entails a microfoundation for macroeconomic models with, for example, Hansen's and Sargent's (2001) multiplier preferences.

What is needed to arrive at these results is a theory of collective decision making with respect to (continuous) convex risk measures. The main clue will be to identify (negated) continuous convex risk measures with their decision-theoretic counterparts, viz. variational preferences, (via their maxmin-expected-utility-plus-penalty representation) and to apply recent results from abstract aggregation theory. Convex risk measures can be represented as negated maxmin expected utility functions with additive convex lower semi-continuous penalty (Föllmer and Schied 2002, 2004), which in turn are in a one-to-one correspondence with the set of so-called variational preference relations (Maccheroni et al. 2006). Given such an individual decision-theoretic foundation for convex risk measures, it is only natural to study the aggregation problem for convex risk measures as an aggregation problem for variational preference relations.

The original, Arrovian preference aggregation theory [originating with Arrow's (1963) famous impossibility theorem; for concise proofs, cf. Fishburn (1970) and $\mathrm{Yu}$ (2012)] does not provide suitable methods to study the aggregation of variational preferences. Even the assumptions in the rich literature on Arrovian social choice 
on economic domains_-cf. e.g. Maskin (1976, 1979), Kalai et al. (1979) or Moulin (1980) for important early contributions as well as Le Breton and Weymark (2002, 2010) and Bossert and Weymark (2006) for more recent work and surveys - are too restrictive for the purposes of the present paper. [For instance, many contributions in this body of literature require the alternatives to form a non-negative orthant and the population to be finite; the only theorem in this literature that might be considered a special case of our results in this paper is the analogue of Arrow's impossibility theorem for expected-utility preferences which Le Breton proved in his habilitation thesis Le Breton (1986), cf. also Le Breton and Weymark (2010).]

That said, there is also a ("non-Arrovian") literature on the aggregation of (generalisations of) expected-utility preferences. This body of literature originated with Harsanyi's (1955) paper on the aggregation of von Neumann and Morgenstern (1944) expected-utility preferences and has seen numerous contributions by diverse other authors. For example, the Paretian aggregation of subjective expected-utility preferences has been studied (motivated by a Bayesian view of probabilities) by, e.g. Hylland and Zeckhauser (1979), Seidenfeld et al. (1989), and Mongin (1995). Gilboa et al. (2004) even generalise Harsanyi's (1955) and Mongin's (1995) characterisations (of social welfare functions as convex combinations of individual utility functions) to a setting where both the utility functions and the probability measures are subjective. Furthermore, Mongin (1998) investigated the aggregation of state-dependent expected-utility preferences, while the aggregation of an even richer class of preference orderings [that includes the Gilboa and Schmeidler (1989) maxmin expected-utility preferences] has been studied quite recently by Gajdos et al. (2008). For all its merits, this body of literature generally does not impose Arrow's (1963) axiom of independence of irrelevant alternatives (let alone systematicity) and, therefore, should not be considered a branch of Arrovian aggregation theory; in addition, the contributions in this literature typically only consider the case of finitely many individuals. In contrast, the present paper does assume the systematicity axiom (a stronger sibling of the independence axiom), thus placing itself more firmly within the Arrovian current of aggregation theory, and studies the case of infinite electorates as well.

The scope of Arrovian aggregation theory has developed considerably during the past decade. It now encompasses aggregation problems of very general form, including even the aggregation of general logical propositions. This area, known as judgment aggregation theory or abstract aggregation theory, has seen seminal contributions by List and Pettit (2002), Dietrich and Mongin (2007), Nehring and Puppe (2007), Dokow and Holzman (2010), Dietrich and List (2007, 2008, 2010); for a survey, see List and Puppe (2009). A very recent development in this area is the investigation of the aggregation of more general propositional attitudes which allows for a unified treatment of both judgment aggregation and probabilistic opinion pooling (cf. McConway (1981) for a seminal contribution to the latter area), cf. Dietrich and List (2010).

One of the recent and less well-known generalisations of classical (Arrovian) preference aggregation theory within judgment aggregation theory is concerned with the aggregation of relational structures (model aggregation). This approach can best be seen as a continuation of Lauwers and Van Liedekerke's far-sighted paper Lauwers and Van Liedekerke (1995) and was elaborated systematically recently by Herzberg 
and Eckert (2012a,b). ${ }^{1}$ It is a rather natural methodological choice to employ model aggregation theory in our analysis of variational preference aggregation, on account of the intrinsic emphasis which model aggregation theory lays on semantics (in comparison with most of the judgment aggregation literature) and also because of its historical roots in preference aggregation theory through the work of Lauwers and Van Liedekerke (1995).

In the present paper, the methodology of model aggregation theory will enable us to prove variational analogues of two of the most important (im)possibility theorems of Arrovian social choice theory-those of Arrow and Fishburn-, and to propose a potential analogue of Campbell's theorem in a generalised variational setting. Moreover, it may well be possible to apply the same proof methodology to obtain similar results for multiple priors preferences (which can be represented by coherent risk measures) and perhaps ultimately even for dynamic variational or multiple priors preferences.

The paper is structured as follows: Sect. 2 reviews the axioms and the representation theorem of variational preferences and relates them to convex risk measures. Section 3 proposes a formal framework for an Arrovian aggregation theory of variational preferences, within which Sect. 4 formulates the main (im)possibility results of this paper. Section 5 then describes briefly the ideas behind the proof methodology (model aggregation theory), while Sect. 6 discusses possible extensions and future research.

In an Appendix, we also apply Dietrich and List (2010) account of majority voting to the problem of variational preference aggregation. The fruit is a possibility theorem, but at the cost of considerable and - at least at first sight-rather unnatural restrictions on the domain of the variational preference aggregator.

\section{Variational preferences and convex risk measures}

Consider a finite set $S$, called the set of states of the world, let $X$ be a convex subset of a vector space $Y$ with more than one element, called the set of consequences, let $\mathcal{F}$ be the set of all functions from $S$ to $X$. Then, $\mathcal{F}$ is a convex subset of the vector space $Y^{S}$. Let $\mathcal{F}_{\mathrm{c}}$ be the set of all constant functions from $S$ to $X$. Every element $x \in X$ can be identified with the constant function $s \mapsto x$ in $\mathcal{F}$ and, thus, with an element of $\mathcal{F}_{\mathrm{c}}$.

Let us now introduce axioms for a binary relation $\succsim$ with symmetric part $\sim$ (i.e. $f \sim g$ if and only if $f \succsim g$ and $g \succsim f$ ) and asymmetric part $\succ$ (i.e. $f \succ g$ if and only if $f \succsim g$ but $g \nsucc f$ ); our formulation of the axioms is borrowed from Maccheroni et al. (2006, p. 1453).

Definition 1 A binary relation $\succsim$ on $\mathcal{F}$ with symmetric part $\sim$ and asymmetric part $\succ$ is a variational preference ordering or convex risk-preference ordering if and only if it satisfies all of the following axioms:

(A1) Weak order properties For all $f, g \in \mathcal{F}$, either $f \succsim g$ or $g \succsim f$ (completeness); for all $f, g, h \in \mathcal{F}$, if $f \succsim g$ and $g \succsim h$, then $f \succsim h$ (transitivity).

\footnotetext{
1 For another recent application of that approach - to the problem of representative-agent microfoundations for certain parametrised aggregator domains_-see Herzberg (2010).
} 
(A2) Weak certainty independence For all $f, g \in \mathcal{F}, x, y \in \mathcal{F}_{\mathrm{c}}$ and $\alpha \in(0,1)$, if

$$
\alpha f+(1-\alpha) x \succsim \alpha g+(1-\alpha) x
$$

then

$$
\alpha f+(1-\alpha) y \succsim \alpha g+(1-\alpha) y \text {. }
$$

(A3) Continuity For all $f, g, h \in \mathcal{F}$, the sets

$$
\{\beta \in[0,1]: \beta f+(1-\beta) g \succsim h\}
$$

and

$$
\{\beta \in[0,1]: h \succsim \beta f+(1-\beta) g\}
$$

are closed.

(A4) Monotonicity For all $f, g \in \mathcal{F}$, if $f(s) \succsim g(s)$ for all $s \in S$, then $f \succsim g$.

(A5) Uncertainty aversion For all $f, g \in \mathcal{F}$ and $\alpha \in(0,1)$, if $f \sim g$, then $\alpha f+(1-$ $\alpha) g \succsim f$

(A6) Non-degeneracy There exist $f, g \in \mathcal{F}$ such that $f \succ g$.

Remark 2 Let $\succsim$ be a binary relation on $\mathcal{F}$ with symmetric part $\sim$ and asymmetric part $\succ$.

1. If $\succsim$ satisfies completeness (A1a), then

$$
f \succsim g \Leftrightarrow f \nprec g
$$

for all $f, g \in \mathcal{F}$.

2. If $\succsim$ satisfies completeness (A1a), then $\succsim$ satisfies continuity (A3) if and only if for all $f, g, h \in \mathcal{F}$ and all $\beta \in[0,1]$, there exist $\alpha, \gamma \in[0,1]$ such that

- $(\alpha, \gamma) \subseteq\{\delta \in[0,1]: \delta f+(1-\delta) g \succsim h\}$ if $\beta f+(1-\beta) g \succsim h$, and

- $(\alpha, \gamma) \subseteq\{\delta \in[0,1]: h \succsim \delta f+(1-\delta) g\}$ if $h \succsim \beta f+(1-\beta) g$,

while either

- $0 \leq \alpha<\beta<\gamma \leq 1$ or

- $0=\alpha=\beta<\gamma \leq 1$ or

- $0 \leq \alpha<\beta=\gamma=1$.

The identification of variational preference relations with convex risk-preference orderings can be justified as follows: on the one hand, Maccheroni et al. (2006, pp. 1453, 1456) have extended the previous work by Gilboa and Schmeidler (1989) and established that a relation $\succsim$ satisfying axioms (A1-A6) allows for a representation in terms of a maxmin expected utility function with additive convex lower semi-continuous penalty: a binary relation $\succsim$ on $\mathcal{F}$ is a variational preference relation if and only if there exists a non-zero linear function $u: X \rightarrow \mathbb{R}$ and a convex 
lower semi-continuous function $c: \Delta \rightarrow[0,+\infty]$ ( $\Delta$ being the set of all probability measures on $S$ ) whose infimum is $>-\infty$ such that for any $f, g \in \mathcal{F}$, one has

$$
f \succsim g \Leftrightarrow \min _{p \in \Delta}\left(\int u \circ f \mathrm{~d} p+c(p)\right) \geq \min _{p \in \Delta}\left(\int u \circ g \mathrm{~d} p+c(p)\right) .
$$

On the other hand, Föllmer and Schied (2002, 2004) have demonstrated that continuous convex risk measures can be represented as negated maxmin expected utility functions with additive convex lower semi-continuous penalty and "real consequences" (i.e. $X \subseteq \mathbb{R}$ ), and vice versa. Therefore, variational preference relations are the ordinal equivalents of continuous convex risk measures.

In our investigation of aggregation of variational preference orderings (i.e. convex risk-preference orderings), it will be helpful to have a more "quantitative" notion of continuity at hand, to distinguish degrees of continuity. For this purpose, we introduce the notion of a witness to continuity. The following definition of being a "witness to continuity" is motivated by the role which the scalars $\alpha, \gamma$ play in the equivalent characterisation of continuity in Remark 2.

Remark 3 Preference orderings that satisfy all variational axioms except continuity nevertheless represent convex risk measures; for such preference orderings, most of the analysis in the present paper still goes through, even in simplified form. Analogues of Arrow's impossibility theorem and Fishburn's possibility theorem still hold in that setting, which corresponds to the aggregation of (not necessarily continuous) convex risk measures. If one is only interested in aggregating (not necessarily continuous) convex risk measures, one may ignore the continuity conditions that follow-at the price of relinquishing an analogue of Campbell's impossibility theorem.

Definition 4 Let $f, g, h \in \mathcal{F}$ and $\beta \in[0,1]$. A pair of real numbers $(\alpha, \gamma) \in[0,1]^{2}$ is called a witness-pair to the continuity of $\succsim$ along $f, g, h \in \mathcal{F}$ in $\beta$ if and only if for all $\delta \in(\alpha, \gamma)$, one has

- $\delta f+(1-\delta) g \prec h$ if $\beta f+(1-\beta) g \prec h$ and

- $h \prec \delta f+(1-\delta) g$ if $h \prec \beta f+(1-\beta) g$,

whilst either

- $\alpha<\beta<\gamma$ or

- $0=\alpha=\beta<\gamma$ or

- $\alpha<\beta=\gamma=1$.

A real number $\varepsilon \in[0,1]$ is called a witness to the continuity of $\succsim$ along $f, g, h \in \mathcal{F}$ in $\beta$ if and only if there exists some $\alpha \in[0,1]$ or $\gamma \in[0,1]$ such that either $(\alpha, \varepsilon)$ or $(\gamma, \varepsilon)$ is a witness-pair to the continuity of $\succsim$ along $f, g, h \in \mathcal{F}$ in $\beta$.

With this definition, we can now rephrase Remark 2:

Remark 5 If $\succsim$ satisfies completeness (A1a), then $\succsim$ satisfies continuity (A3) if and only if for all $f, g, h \in \mathcal{F}$ and all $\beta \in[0,1]$ there exists a witness to the continuity of $\succsim$ along $f, g, h \in \mathcal{F}$ in $\beta$. 


\section{Aggregation of variational preferences}

Consider a set $N$ (finite or infinite), which we shall call population or electorate. Elements of $N$ are called individuals, subsets of $N$ are called coalitions. Suppose that each individual $i \in N$ is endowed with a variational preference ordering $\succsim_{i}$ (as defined in Sect. 2), any such resulting $N$-sequence $\succsim=\left(\succsim_{i}\right)_{i \in N}$ is called a variational preference profile. In various circumstances-for instance, in the course of making certain policy choices - the question will arise whether one can aggregate the individual variational preference orderings and obtain a social variational preference ordering (i.e. an aggregate of the individual variational preferences $\succsim_{i}$ which itself happens to be variational preference relation). And if so, are there any rules, satisfying certain rationality conditions, which can be used to assign a (social) variational preference ordering to all variational preference profiles — or at least to a large class of variational preference profiles?

We shall show that any such rule whose domain encompasses a rich class of variational preference profiles must be dictatorial in the case of finite $N$ and, thus, establish an equivalent of Arrow's (1963) impossibility theorem for variational preference aggregation. For the case of infinite $N$, we shall prove a possibility result for infinite $N$ under the assumption that the aggregator domain contains only equicontinuous variational preference profiles; this result can be seen as an variational-preference analogue of Fishburn's (1970) possibility theorem. We shall also outline how a potential analogue of Campbell's impossibility theorem may be obtained.

As we shall see in an appendix, on certain restricted domains of profiles for finite electorates, the majority voting rule-which also satisfies two important rationality axioms — can be used to obtain a social variational preference ordering.

\section{Main results: variational preference aggregation for rich aggregator domains}

Denote the set of all variational preference relations on $\mathcal{F}$ by $\mathcal{P}$.

In this paper, a pre-variational preference aggregator is a map $F$ with domain $\operatorname{dom}(F) \subseteq \mathcal{P}^{N}$ whose range is a set of complete binary relations on $\mathcal{F}$. A strictly variational preference aggregator or convex risk-preference aggregator is a map $F$ from a subset $\operatorname{dom}(F) \subseteq \mathcal{P}^{N}$ to $\mathcal{P}$. A pre-variational preference aggregator $F$ is said to be

- universal if and only if $\operatorname{dom}(F)=\mathcal{P}^{N}$ (so that $F: \mathcal{P}^{N} \rightarrow \mathcal{P}$ );

- weakly universal if and only if $\operatorname{dom}(F)$ is a rich aggregator domain. Herein, a subset $\mathbb{D} \subseteq \mathcal{P}^{N}$ is called a rich aggregator domain if and only if there are $f, f^{\prime}, g, g^{\prime} \in \mathcal{F}$ and variational preference orderings $\succsim_{1}, \succsim_{2}, \succsim_{3}$ such that

$-f \succsim_{1} g, f^{\prime} \succsim_{1} g^{\prime}, \quad f \succsim_{2} g, f^{\prime} \prec_{2} g^{\prime}, \quad f \prec_{3} g, f^{\prime} \succsim_{3} g^{\prime}$, and

- $\left\{\succsim_{1}, \succsim_{2}, \succsim_{3}\right\}^{N} \subseteq \mathbb{D}$;

- systematic if and only if for every $\succsim \in \operatorname{dom}(F)$ and all $f, f^{\prime}, g, g^{\prime} \in \mathcal{F}$ with $\left\{i \in N: f \succsim_{i} g\right\}=\left\{i \in N: f^{\prime} \succsim_{i} g^{\prime}\right\}$ one has

$$
f F(\succsim) g \Leftrightarrow f^{\prime} F(\succsim) g^{\prime}
$$


- Paretian if and only if for every $\succsim \in \operatorname{dom}(F)$ and all $f, g \in \mathcal{F}$, if $f \succsim_{i} g$ for all $i \in N$, then $f F(\succsim) g$;

- dictatorial if and only if there exists some $i \in N$ (called dictator) such that for every $\succsim \in \operatorname{dom}(F)$ and all $f, g \in \mathcal{F}$,

$$
f F(\succsim) g \Leftrightarrow f \succsim_{i} g
$$

The modification "weakly" in "weakly universal" is justified:

Remark 6 If $S$ contains at least two elements, then $\mathcal{P}^{N}$ is a rich aggregator domain, and every universal aggregator is also weakly universal.

(All proofs can be found in Appendix C.) Clearly, every dictatorial $F$ can be extended to a universal, systematic and Paretian aggregator. It is remarkable that even the converse holds true.

Theorem 7 Let $N$ be finite and let $F$ be a pre-variational preference aggregator. $F$ is weakly universal, systematic and Paretian if and only if it is dictatorial.

(Theorem 7 is the variational preference analogue of Arrow's (1963) possibility theorem.)

One can obtain a possibility result for infinite $N$ by demanding equicontinuity of all variational preference profiles in the aggregator domain - rather than mere continuity of the preferences in all variational preference profiles. A profile $\succsim=\left(\succsim_{i}\right)_{i \in N}$ is said to be continuous if and only if $\succsim_{i}$ is continuous for all $i \in \bar{\tau}$; it is said to be equicontinuous if and only if for all $f, g, h \in \mathcal{F}$ and all $\beta \in[0,1]$, there exist $\alpha, \gamma \in[0,1]$ which for all $i \in N$ are a witness-pair to the continuity of $\succsim_{i}$ along $f, g, h$ in $\beta$.

Theorem 8 Let $N$ be an infinite set, and let $\mathbb{D} \subseteq \mathcal{P}^{N}$ be a rich aggregator domain such that all profiles in $\mathbb{D}$ are equicontinuous. Then there exist non-dictatorial, weakly universal, systematic and Paretian strictly variational preference aggregators $F$ : $\mathbb{D} \rightarrow \mathcal{P}$.

(Theorem 8 is the variational preference analogue of Fishburn's (1970) possibility theorem. The condition of equicontinuity means that every profile in the aggregator domain consists of preferences that have the same modulus of continuity.)

Remark 9 For the case of infinite electorates, one can obtain an analogue of Campbell's (1990) impossibility theorem as follows. Let $\mathcal{Q}$ denote the set of Monotonic Bernoullian Archimedean (MBA) preferences recently introduced by Cerreia-Vioglio et al. (2011). Note that the Archimedean property is of the form $\forall f, g, h \exists \alpha, \beta \ldots$ where ... is a quantifier-free formula, therefore, any aggregator from $\mathcal{Q}^{N}$ to $\mathcal{Q}$ will have to preserve a $\forall \exists$ formula. Now, for infinite $N$, it will-in non-degenerate casesnot be difficult to construct profiles for which the witnesses $\alpha, \beta \in(0,1)$ of the Archimedean property for fixed acts $f, g, h$ will converge to the extreme values 1 and 0 , respectively. This implies_-by a reasoning analogous to that in Herzberg and Eckert 
(2012b, Theorem 4.1$)^{2}$ - that any universal and Paretian aggregator from $\mathcal{Q}^{N}$ to $\mathcal{Q}$ will be a dictatorship.

Again using the model-theoretic approach to aggregation [cf. Herzberg and Eckert (2012b)], one can also obtain an analogue of Arrow's impossibility theorem for the aggregation of finite profiles of MBA preferences. In addition, it might be possible to establish an analogue of Fishburn's possibility theorem which asserts the possibility of aggregating infinite profiles of MBA preferences if one admits in the aggregator domain only_what may be called-equi-Archimedean profiles, viz. sequences of preference relations that have a uniform pair of witnesses to the Archimedean property.

The details of this proposal are worked out in a paper in preparation.

\section{Proof idea}

The shortest route in proving the above theorems is to invoke recent results from model aggregation theory, due to Herzberg and Eckert (2012a) who generalised previous findings by Lauwers and Van Liedekerke (1995). To employ these results, one needs to reformulate the variational preference aggregation problem as a model aggregation problem (see Appendix B); thereafter, the proofs follow relatively easily from the model aggregation theory in Herzberg and Eckert (2012a) (see Appendix C). In this section, we briefly describe model aggregation theory and its application to the aggregation of variational preferences; a rigorous review can be found in Appendix A.

Model aggregation theory studies the aggregation of first-order structures (in the sense of mathematical logic). An aggregator in this setting is then just a map from a set of $N$-sequences of structures of a certain type to a set of structures of such type. It is not difficult to formulate analogues of Arrow's (1963) rationality assumptions in this framework.

Of utmost importance is the notion of a decisive coalition with respect to an aggregator $F$. A coalition $D$ is said to be decisive with respect to an aggregator $F$ if and only if it can be written in the form $D=\left\{i \in N:\left(\mathcal{F}, \succsim_{i}\right) \models \phi\right\}$ for some profile $\succsim \in \operatorname{dom}(F)$ and some quantifier-free formula $\phi$ such that $(\mathcal{F}, F(\succsim)) \models \phi$.

Denoting the set of all decisive coalitions with respect to $F$ by $\overline{\mathcal{D}}_{F}$, one can next prove the following key lemma:

Lemma 10 If $F$ is a weakly universal, systematic and Paretian strictly variational preference aggregator, then $\mathcal{D}_{F}$ is an ultrafilter on $N .^{3}$

\footnotetext{
${ }^{2}$ It is important to note that, as the proof of that result Herzberg and Eckert (2012a, Theorem 4.1) reveals, the condition on formula $\phi$ described in the second part of the theorem is also assumed in the first part, even though the theorem does not state this explicitly.

3 An ultrafilter on $N$ is a non-empty set $\mathcal{D}$ of coalitions which is not equal to the powerset of $N$, is closed under supersets (i.e. if $D \in \mathcal{D}$ and $D^{\prime} \supseteq D$, then $D^{\prime} \in \mathcal{D}$ ), closed under intersections (i.e. if $D, D^{\prime} \in \mathcal{D}$, then $D \cap D^{\prime} \in \mathcal{D}$ ) and has the property that for any coalition $D$ either $D \in \mathcal{D}$ or $N \backslash D \in \mathcal{D}$. A filter on $N$ is a set of coalitions that has the first three properties, but may lack the last one. One can show that ultrafilters are nothing else but $\subseteq$-maximal filters.
} 
The proof of Lemma 10 uses a slight generalisation of the main lemma in Lauwers and Van Liedekerke (1995, Lemma 2): ${ }^{4}$ In the proof of that lemma, the ultrafilter properties (non-triviality, closure under supersets and intersections, dichotomy) are verified by constructing appropriate profiles through exploiting the richness of the aggregator domain.

Since ultrafilters on finite sets are always principal (i.e. systems of supersets of singletons), Lemma 10 quickly leads to a proof of the "only if" part in Theorem 7. The proof of the "if" part in Theorem 7 is straightforward.

Using the ultrafilter property of the set of decisive coalitions, Theorem 8 can now be proved through applications of Łoś's theorem: for, one can apply Lemma 10 to show that any weakly universal, systematic and Paretian pre-variational preference aggregator $F$ maps every variational preference profile to the restriction (to the original domain $\mathcal{F}$ ) of its ultraproduct (with respect to the ultrafilter $\mathcal{D}_{F}$ of decisive coalitions), and conversely, Łoś's theorem implies that every pre-variational preference aggregator $F$ which assigns to each variational preference profile in $\operatorname{dom}(F)$ the restriction of its ultraproduct with respect to a fixed ultrafilter $\mathcal{D}$ constitutes a systematic Paretian pre-variational preference aggregator (which is weakly universal if $\operatorname{dom}(F)$ is a rich aggregator domain). Now, since-again by Łoś's theorem-restricted ultraproducts preserve universal formulae (also sometimes called $\Pi_{1}$ formulae) that hold in all factor structures, it is clear that the aggregate of an equicontinuous variational preference profile under a weakly universal systematic Paretian pre-variational preference aggregator must again be continuous and, thus, a variational preference profile. Hence, every weakly universal, systematic, Paretian pre-variational preference aggregator whose domain only consists of equicontinuous variational preference profiles is actually a strictly variational preference aggregator. Now, for infinite $N$, there exist non-principal ultrafilters $\mathcal{U}$ on $N$. Choose such a $\mathcal{U}$ and let $F: \mathbb{D} \rightarrow \mathcal{P}$ be a map whose domain only contains equicontinuous variational preference profiles and which assigns to each element of $\mathbb{D}$ the restriction of its ultraproduct with respect to $\mathcal{U}$. This $F$ will then be a strictly variational preference aggregator which is not dictatorial, establishing Theorem 8 .

\section{Possible extensions and future research}

Using the methodology of the present paper, one can also study the aggregation of coherent risk measures for a finite set of states of the world. For, coherent risk measures can be written as negated maxmin expected utility functions, which in turn represent multiple priors preferences, as shown by Gilboa and Schmeidler (1989). (For recent results on maxmin expected utility functions, see e.g. de Castro and Chateauneuf (2011) and the references therein.) Hence, the aggregation of coherent risk measures can be reformulated as an aggregation problem for certainty-independent, continuous, monotonic, uncertainty-averse and non-degenerate weak orders, and the theory of model aggregation can again be used to prove impossibility and possibility results. It

\footnotetext{
4 Whilst the published proof of Lauwers's and Van Liedekerke's (1995) lemma is incomplete, an addendum by Herzberg et al. (2010) has recently filled the gap.
} 
might perhaps even be possible to employ the methodology of the present paper to study the Arrovian aggregation of Choquet expected utility preferences [cf. Chateauneuf et al. (2003)] or neo-additive Choquet preferences [cf. Chateauneuf et al. (2007) and for more recent work e.g. Dominiak and Lefort (2012)].

Also, variational preferences or Choquet expected utility preferences (let alone multiple priors preferences) do not exhaust all possibilities of modelling Ellsberg (1961) ambiguity (i.e. uncertainty in the sense of Knight (1921); see Ellsberg et al. (2011) as well as Nau (2011) for some history of the literature on ambiguity). Even though this would mean leaving aside the original motivation of aggregating risk measures, a systematic aggregation theory for preferences from more general classes of preferences encoding ambiguity, in particular the MBA class of Cerreia-Vioglio et al. (2011), is an interesting research project and will be addressed in the future.

Another possible domain of application of the approach in this paper might be an Arrovian aggregation theory of dynamic variational preferences and, thus, of dynamic convex risk measures. For, the representation theorem of Föllmer and Schied (2002, 2004) has been extended to a dynamic setting by Detlefsen and Scandolo (2005) in a paper on dynamic convex risk measures which builds upon on Riedel (2004) seminal article on dynamic coherent risk measures. Moreover, Maccheroni et al. (2006) have recently developed a dynamic generalisation of their axiomatisation of variational preferences Maccheroni et al. (2006). Combining their theorem with Detlefsen and Scandolo's result, one obtains a decision-theoretic foundation of dynamic convex risk measures in terms of dynamic variational preferences.

Furthermore, the systematicity condition can possibly be relaxed. For, systematicity is equivalent to the weaker aggregator condition of independence if the conditional entailment relation among the set of test sentences has full transitive closure.

Finally, it would be desirable to allow for infinitely many, not just finitely many, states of the world. However, a straightforward generalisation of our arguments to this infinitary setting would then need to refer to conjunctions of infinitely many propositions. This might be achieved by a generalisation of our present methodology (model aggregation) based on the model theory for infinitary logic, cf. e.g. Keisler (1971) (possibly the work of Waszkiewicz and Weglorz (1969) might also be employed to formulate analogues of the ultraproduct construction for infinitary logic, cf. the review of that work by Feferman in Mathematical Reviews 41 \#8220).

An entirely different approach to the aggregation of variational preferences would be the analysis of the (in general inconsistent, cyclical) aggregates of variational preferences which an application of the majority rule would yield. Results about utility representations of certain binary relations on topological vector spaces (cf. Neuefeind and Trockel (1995)) might prove useful for that purpose.

\section{Conclusion}

We have formulated Arrow-type aggregation problems for convex risk measures or variational preferences. Choosing a methodology inspired by Lauwers and Van Liedekerke (1995), one can prove analogues of Arrow's impossibility theorem and Fishburn's possibility theorem (and with some modifications even a potential analogue of Campbell's impossibility theorem). The proof method is sufficiently general to be applied to 
Arrow-type aggregation of coherent risk measures or multiple priors preferences, and perhaps even dynamic convex or dynamic coherent risk measures and their variational counterparts.

The results can, for instance, be used for the constitutional design of panels consisting of several risk managers. In light of the variational analogue of Arrow's impossibility theorem, the panel should not assess financial risks ad hoc; rather, it should agree on a joint risk measure from the outset.

Another application is in the area of macroeconomic theory: the variational analogue of Fishburn's theorem provides a microfoundation for social preferences that are variational, e.g. Hansen's and Sargent's (2001) multiplier preferences.

\section{Appendix A: Review of model aggregation theory}

The theory of model aggregation was begun by Lauwers and Van Liedekerke (1995) (see also Herzberg et al. (2010)) and continued recently by Herzberg and Eckert (2012a,b). In the following, we only review special cases of the most important known results from model aggregation with particular relevance for the analysis of variational preference aggregation. In fact, the presentation in this appendix is only slightly more general than the work of Lauwers and Van Liedekerke (1995) - in that it allows for arbitrarily many predicate symbols rather than just one-, whence any reader who knows the work of Lauwers and Van Liedekerke (1995) may well skip this appendix. For proofs and a more general account of model aggregation, see Herzberg and Eckert (2012a,b).

We assume in this section that the reader has some basic knowledge of model theory. The paper by Lauwers and Van Liedekerke (1995) contains a short introduction to logic and model theory for social choice theorists; more comprehensive introductions can be found in textbooks such as those by Bell and Slomson (1969) or Hodges (1985).

Let $A$ be a set. Let $\mathcal{L}$ be a language consisting of predicate symbols $\dot{P}_{n}, n \in \kappa$, and constant symbols $\dot{a}$ for all elements $a$ of $A$. The arity of $\dot{P}_{n}$ will be denoted $\delta(n)$, for all $n \in \kappa$.

For the purposes of this paper, an $\mathcal{L}$-structure is a pair $\mathfrak{B}=\left\langle B,\left\langle P_{n}^{\mathfrak{B}}\right\rangle_{n \in \kappa}\right\rangle$ consisting of a set $B \supseteq A$ (called the domain of $\mathfrak{B}$ ) and certain sets $P_{n}^{B} \subseteq B^{\delta(n)}$ which serve to interpret the predicate symbols $\dot{P}_{n}$ through Tarski's definition of truth. We require that, by definition any $\mathcal{L}$-structure interprets the constant symbols $\dot{a}$ canonically, i.e. by $a$, for any $a \in A$.

Let $\mathcal{S}$ be the set of atomic formulae in $\mathcal{L}$. Let $\mathcal{T}$ be the Boolean closure of $\mathcal{S}$, i.e. the closure of $\mathcal{S}$ under the logical connectives $\dot{\neg}, \dot{\wedge}, \dot{V}$. The elements of $\mathcal{T}$ are called test sentences, and the elements of $\mathcal{S}$ are called basic test sentences.

Let $T$ be a consistent set of universal sentences in $\mathcal{L} .^{5}$, and let $\Omega$ be the collection of models of $T$ with domain $A$. As is usual in model theory, the restriction of an $\mathcal{L}$-structure $\mathfrak{B}$ is the $\mathcal{L}$-structure that is obtained by restricting the interpretations of the relation symbol to the domain $A$; it is denoted $\operatorname{res}_{A} \mathfrak{B}$.

\footnotetext{
5 A sentence is universal if it (in its prenex normal form) has the form $\left(\dot{\forall} \dot{v}_{k_{1}} \dot{ }\right) \cdots\left(\dot{\forall} \dot{v}_{k_{m}}\right) \phi$ for some formula $\phi$ that does not contain any quantifiers and some non-negative integer $m$.
} 
We assume that there are two sentences in $\mathcal{S}$, henceforth denoted $\mu, v \in \mathcal{S}$, such that each of $\mu \dot{\wedge} \nu, \mu \dot{\wedge} \dot{\neg} v$ and $\dot{\neg} \mu \dot{\wedge} v$ is consistent with $T$, in symbols,

$$
T \cup\{\mu \dot{\wedge} \nu\} \not \forall \perp, \quad T \cup\{\mu \dot{\wedge} \dot{\neg} \nu\} \forall \perp, \quad T \cup\{\dot{\neg} \mu \dot{\wedge} \nu\} \not \perp
$$

(wherein $\perp$ is shorthand for $\phi \dot{\wedge} \dot{\neg} \phi$ for some sentence $\phi$ ).

Since $\mathcal{S}$ is the set of all atomic formulae in $\mathcal{L}$ and $T$ is a set of universal sentences, the following propositions hold for all $\mathcal{L}$-structures $\mathfrak{A}$ and all $\mathfrak{A}_{1}, \mathfrak{A}_{2} \in \Omega$ :

$$
\begin{aligned}
& \left(\forall \lambda \in \mathcal{S} \quad\left(\mathfrak{A}_{1} \models \lambda \Leftrightarrow \mathfrak{A}_{2} \models \lambda\right)\right) \Rightarrow \mathfrak{A}_{1}=\mathfrak{A}_{2} . \\
& \mathfrak{A} \models T \Rightarrow \operatorname{res}_{A} \mathfrak{A} \in \Omega \\
& \forall \lambda \in \mathcal{T} \quad\left(\mathfrak{A} \models \lambda \Leftrightarrow \operatorname{res}_{A} \mathfrak{A} \models \lambda\right) .
\end{aligned}
$$

Elements of $\Omega^{N}$ will be called profiles. An aggregator is a map $f$ whose domain $\operatorname{dom}(f)$ is a subset of $\Omega^{N}$ and whose range is a subset of $\Omega{ }^{6}$

For all $\lambda \in \mathcal{T}$ and all $\omega \in \Omega^{N}$, we denote the coalition supporting $\lambda$ given profile $\omega$, by

$$
C(\omega, \lambda):=\left\{i \in N: \omega_{i} \models \lambda\right\} .
$$

Let us fix an aggregator $f$. Consider the following axioms:

(A1). $\operatorname{dom}(f)=\Omega^{N}$.

(A1'). There exist models $\mathfrak{A}_{1}, \mathfrak{A}_{2}, \mathfrak{A}_{3} \in \Omega$ such that

1. $\mathfrak{A}_{1} \models \mu \dot{\wedge} v, \mathfrak{A}_{2} \models \mu \dot{\wedge} \dot{\sim} v, \mathfrak{A}_{3} \models \dot{\neg} \mu \dot{\wedge} v$, and

2. $\left\{\mathfrak{A}_{1}, \mathfrak{A}_{2}, \mathfrak{A}_{3}\right\}^{N} \subseteq \operatorname{dom}(f)$.

(A2). For all $\omega \in \operatorname{dom}(f)$ and all $\lambda \in \mathcal{T}$, if $f(\omega) \models \lambda$, then $C(\omega, \lambda) \neq \emptyset$.

(A3). For all $\omega, \omega^{\prime} \in \operatorname{dom}(f)$ and all $\lambda, \lambda^{\prime} \in \mathcal{T}$ such that $C(\omega, \lambda)=C\left(\omega^{\prime}, \lambda^{\prime}\right)$, one has $f(\omega) \models \lambda$ if and only if $f\left(\omega^{\prime}\right) \models \lambda^{\prime}$.

(A1) is the axiom of universality. Axiom (A2) is a generalised Pareto Principle. (A3) is a generalised form of the axiom of systematicity, which itself is a strong variant of the axiom of Independence of Irrelevant Alternatives. ${ }^{7}$

By our assumptions on $\mu, v \in \mathcal{S}$, there must be $\mathcal{L}$-structures $\mathfrak{A}_{1}, \mathfrak{A}_{2}, \mathfrak{A}_{3}$ such that $\mathfrak{A}_{1}, \mathfrak{A}_{2}, \mathfrak{A}_{3} \models T$ as well as $\mathfrak{A}_{1} \models \mu \dot{\wedge} v, \mathfrak{A}_{2} \models \mu \dot{\wedge} \dot{\sim} v, \mathfrak{A}_{3} \models \dot{\sim} \mu \dot{\wedge} v$. Since $T$ is universal and so are all elements of $\mathcal{T}$, we may assume that $\mathfrak{A}_{1}, \mathfrak{A}_{2}, \mathfrak{A}_{3}$ all have

\footnotetext{
6 We deviate from Lauwers's and Van Liedekerke's (1995) notation as follows:

- Aggregators will be denoted by $f$ (instead of AF).

- Profiles will be denoted by $\omega$ or $\left\langle\omega_{i}: i \in N\right\rangle$ (instead of $\left\langle\mathcal{A}_{i}: i \in N\right\rangle$ ).

- The image of a profile $\omega$ under an aggregator $f$ will be denoted by $f(\omega)$ (instead of $\mathcal{A}(\omega)$ ).
}

7 Systematicity vacuously implies Independence of Irrelevant Alternatives. The converse is true under additional hypotheses. In the preference aggregation framework, the combination of Independence of Irrelevant Alternatives and the Pareto Principle implies Systematicity, if the individual preferences are complete and quasi-transitive [cf. Lauwers and Van Liedekerke (1995, Section 6, p. 232)]. 
domain $A$ (otherwise, take their restriction to $A$ ). Hence, Axiom (A1') is simply a weak version of (A1) because of our assumption about the sentences $\mu, v \in \mathcal{S}$.

Given an aggregator $f$, we define the set of decisive coalitions by

$$
\mathcal{D}_{f}:=\{C(\omega, \lambda): \omega \in \operatorname{dom}(f), \quad \lambda \in \mathcal{T}, \quad f(\omega) \models \lambda\} .
$$

It is not difficult to verify that systematic aggregators are characterised by their sets of decisive coalitions:

Remark 11 If $f$ satisfies (A3), then for all $\omega \in \operatorname{dom}(f)$ and $\lambda \in \mathcal{T}$,

$$
C(\omega, \lambda) \in \mathcal{D}_{f} \Leftrightarrow f(\omega) \models \lambda .
$$

This framework is sufficiently general to cover the cases of preference aggregation, propositional judgment aggregation, and modal aggregation. ${ }^{8}$ The general model aggregation theory in Herzberg and Eckert (2012a,b) admits more general sets of test sentences $\mathcal{T}$ and relaxes the aggregator axioms (A2) and (A3).

The key result of model aggregation is the following lemma: ${ }^{9}$

Lemma 12 Let $f$ be weakly universal, systematic, and Paretian. Then, $\mathcal{D}_{f}$ is an ultrafilter. $^{10}$

We say that $f$ is dictatorial if and only if there exists some $i_{f} \in N$ (called the dictator) such that $\mathcal{D}_{f}=\left\{J \subseteq N: i_{f} \in J\right\}$.

Remark 13 Let $f$ be an aggregator, and suppose $N$ is finite. Then, $f$ is dictatorial if and only if $\mathcal{D}_{f}$ is an ultrafilter.

As a corollary of the ultrafilter property of the set of decisive coalitions (see Lemma 12), we then get an analogue of Arrow's impossibility theorem:

Corollary 14 (Impossibility theorem) Let $f$ be weakly universal, systematic, and Paretian. If $N$ is finite, then $f$ is dictatorial.

By Łoś's theorem Los (1955):

Remark 15 If $\mathcal{D}$ is an ultrafilter, then

$$
\operatorname{res}_{A} \prod_{i \in N} \omega_{i} / \mathcal{D} \models \lambda \Leftrightarrow C(\omega, \lambda) \in \mathcal{D}
$$

for all $\omega \in \Omega^{N}$ and $\lambda \in \mathcal{T}$.

\footnotetext{
${ }^{8}$ For example, for preference aggregation, one lets $\mathcal{L}$ have a single binary predicate $\dot{P}$, modelling the preference relation. The set $A$ will be the set of alternatives. The interpretation of $\dot{P}(\dot{a}, \dot{b})$ will be " $a$ is preferred to $b$ ". (Thus, the interpretation of $\omega_{i} \models \dot{P}(\dot{a}, \dot{b})$ is "under profile $\omega$, individual $i$ prefers $a$ to $b$ ", and the interpretation of $f(\omega) \models \dot{P}(\dot{a}, \dot{b})$ is "under profile $\omega, a$ is socially preferred to $b$ ".) $T$ can be any universal theory in that language. For propositional judgment aggregation, one lets $\mathcal{L}$ have a single unary predicate $\dot{B}$, modelling a belief operator. The set $A$ will be the agenda. The interpretation of $\dot{B} \dot{a}$ " $a$ is accepted". (Thus, the interpretation of $\omega_{i} \models \dot{B} \dot{a}$ is "under profile $\omega$, individual $i$ accepts $a$ ", and the interpretation of $f(\omega) \models \dot{B} \dot{a}$ is "under profile $\omega, a$ is socially accepted".) $T$ can be any universal theory in that language.

${ }^{9}$ Lemma 12 slightly generalises the main lemma in Lauwers and Van Liedekerke (1995, Lemma 2); a proof in a more general setting can be found in the first paper of Herzberg and Eckert (2012b).

${ }^{10}$ For the definition of an ultrafilter, see footnote 3 on page 13.
} 
Lemma 16 Let $f$ be weakly universal, systematic, and Paretian, then $f(\omega)=$ $\operatorname{res}_{A} \prod_{i \in N} \omega_{i} / \mathcal{D}_{f}$ for all $\omega \in \operatorname{dom}(f)$.

Lemma 17 Suppose $\mathcal{D}$ is an ultrafilter, and consider the aggregator res $\mathrm{s}_{A} \prod / \mathcal{D}$, defined by

$$
\operatorname{res}_{A} \prod / \mathcal{D}: \Omega^{N} \rightarrow \Omega, \quad \omega \mapsto \operatorname{res}_{A} \prod_{i \in N} \omega_{i} / \mathcal{D}
$$

Then res ${ }_{A} \prod / \mathcal{D}$ is a universal, systematic and Paretian aggregator.

Let $\beta N$ denote the set of all ultrafilters on the set $N$, and let $\mathcal{A R}$ be the set of all universal, systematic and Paretian aggregators. Now one can prove a general version of the Kirman and Sondermann (1972) correspondence: ${ }^{11}$

Theorem 18 (Kirman-Sondermann correspondence) There is a bijection $\Lambda: \mathcal{A R} \rightarrow$ $\beta N$, given by

$$
\forall f \in \mathcal{A R} \quad \Lambda(f)=\mathcal{D}_{f} .
$$

Its inverse is given by

$$
\forall \mathcal{D} \in \beta N \quad \Lambda^{-1}(\mathcal{D})=\operatorname{res}_{A} \prod / \mathcal{D}
$$

wherein, as in Lemma 17, $\operatorname{res}_{A} \prod / \mathcal{D}: \omega \mapsto \operatorname{res}_{A} \prod_{i \in N} \omega_{i} / \mathcal{D}$.

Consider an arbitrary $\mathcal{L}$-sentence which is not universal. In its prenex normal form it can be written as $\left.\psi \equiv \ddot{(} \dot{\forall} \dot{x}_{1}\right) \ldots\left(\dot{\forall} \dot{x}_{m} \ddot{)} \ddot{(} \dot{\exists} \dot{y}\right) \phi\left(\dot{x}_{1}, \ldots, \dot{x}_{m} ; \dot{y}\right)$, wherein $m$ is a nonnegative integer and $\phi\left(\dot{x}_{1}, \ldots, \dot{x}_{m} ; \dot{y}\right)$ is an $\mathcal{L}$-formula with $m+1$ free variables whose logical symbols are conjunctions and existential quantifiers only. For the rest of this section, $\psi$ and $\phi$ are fixed in this manner.

We say that a profile $\omega \in \Omega^{N}$ has finite witness multiplicity with respect to $\psi$ if and only if $\omega_{i} \models \psi$ for all $i \in N$, but for all $a_{1}, \ldots, a_{m}, a^{\prime} \in A$, the coalition $\left\{i \in N: \omega \models \phi\left(a_{1}, \ldots, a_{m} ; a^{\prime}\right)\right\}$ is finite.

An aggregator $f$ is said to preserve an $\mathcal{L}$-sentence $\psi$ if and only if for all $\omega \in$ $\operatorname{dom}(f)$, one has $f(\omega) \models \psi$ whenever $\omega_{i} \models \psi$ for all $i \in N$. We then have the following theorem: ${ }^{12}$

Theorem 19 Let $f$ be weakly universal, systematic and Paretian, suppose $f$ preserves $\psi$, and assume that there exists some $\omega \in \Omega^{I}$ with finite witness multiplicity with respect to $\psi$. Then, $f$ is a dictatorship.

\footnotetext{
11 This Theorem 18 is a slight generalisation of Lauwers and Van Liedekerke's main theorem; its proof-in a more general framework than that of the present paper-can be found in Herzberg and Eckert (2012b).

12 This theorem is in some sense an abstract version of a similar result by Lauwers and Van Liedekerke (1995, p. 230, Property 4); its proof can be found in the second paper of Herzberg and Eckert (2012a).
} 


\section{Appendix B: Variational preference aggregation as model aggregation}

As we have remarked before, our proofs depend largely on the recent results on model aggregation by Herzberg and Eckert $(2012 a, b)$ that generalise previous work by Lauwers and Van Liedekerke (1995). The key to the proof of Theorem 7 is, therefore, the rephrasing of the variational preference aggregation problem in the framework of first-order model theory.

The formulation of the variational preference aggregation problem in the framework of first-order model theory can even be accomplished without appealing to multisorted predicate logic, as it will turn out that one can identify the closed unit interval $[0,1] \subseteq \mathbb{R}$ and the open unit interval $(0,1) \subseteq \mathbb{R}$ with subsets of $\mathcal{F}_{c}$, and hence of $\mathcal{F}$. The domain of the model-theoretic structures to be aggregated will, thus be just $\mathcal{F}$, and individual constant and variable symbols will always be interpreted as referring to constant or variable elements of $\mathcal{F}$.

To embed the closed and open unit intervals of $\mathbb{R}$ into $\mathcal{F}$, choose two distinct elements $x_{0}, x_{1} \in X$, and define for all $\alpha \in[0,1]$ a constant function $\check{\alpha}$ by

$$
\check{\alpha}: s \mapsto \alpha x_{0}+(1-\alpha) x_{1}
$$

Clearly, the map $\alpha \mapsto \check{\alpha}$ is injective. ${ }^{13}$ Hence, if we define

$$
\bar{I}=\{\check{\alpha}: \alpha \in[0,1]\}
$$

and

$$
I=\{\check{\alpha}: \alpha \in(0,1)\}=\bar{I} \backslash\left\{x_{0}, x_{1}\right\}
$$

there is a canonical bijection between $\bar{I}$ and $[0,1] \subseteq \mathbb{R}$ as well as between $I$ and $(0,1) \subseteq \mathbb{R}$.

This allows us to define a mixture operator $m: \bar{I} \times \mathcal{F}^{2} \rightarrow \mathcal{F}$ as follows: For all $\alpha \in[0,1]$ and $f, g \in \mathcal{F}$, put

$$
m(\check{\alpha} ; f, g)=\alpha f+(1-\alpha) g \in \mathcal{F} .
$$

(Recall that $\mathcal{F}$ is a convex subset of the vector space $Y^{S}$.) For every $s \in S$, let $\pi_{s}: \mathcal{F} \rightarrow \mathcal{F}_{c}$ be the projection operator which maps $f$ to the constant function with range $\{f(s)\}$, so that $\pi_{s}(f): s \mapsto f(s)$ for all $f \in \mathcal{F}$.

13 For, if

$$
\alpha x_{0}+(1-\alpha) x_{1}=\beta x_{0}+(1-\beta) x_{1}
$$

for some $\alpha, \beta \in[0,1]$ with $\alpha \neq \beta$, then

$$
(\alpha-\beta) x_{0}=(\alpha-\beta) x_{1}
$$

and thus $x_{0}=x_{1}$, contradiction. 
Finally, one can define a linear ordering $<_{\bar{I}}$ on $\bar{I}$ by

$$
\check{\alpha}<_{\bar{I}} \check{\beta} \Leftrightarrow \alpha<\beta
$$

for all $\alpha, \beta \in[0,1]$.

With these definitions, we may now consider the following axioms for a binary relation $\succsim$ with symmetric part $\sim$ (i.e. $f \sim g$ if and only if $f \succsim g$ and $g \succsim f$ ) and asymmetric part $\succ$ (i.e. $f \succ g$ if and only if $f \succsim g$ but $g \nsucc f$ ):

(A1) Weak order properties For all $f, g \in \mathcal{F}$, either $f \succsim g$ or $g \succsim f$; for all $f, g, h \in \mathcal{F}$, if $f \succsim g$ and $g \succsim h$, then $f \succsim h$.

(A2) Weak certainty independence For all $f, g \in \mathcal{F}, x, y \in \mathcal{F}_{c}$ and $a \in I$, if

$$
m(a ; f, x) \succsim m(a ; g, x)
$$

then

$$
m(a ; f, y) \succsim m(a ; g, y)
$$

(A3) Continuity For all $f, g, h \in \mathcal{F}$ and all $b \in I$, there exist $a, c \in \bar{I}$ such that

- if $m(b ; f, g) \succsim h$, then for all $d \in I$ with $a<_{\bar{I}} d<_{\bar{I}} c$, one has $m(d ; f, g) \succsim h$, and

- if $h \succsim m(b ; f, g)$, then for all $d \in I$ with $a<_{\bar{I}} d<_{\bar{I}} c$, one has $h \succsim m(d ; f, g)$, while either

$-x_{0} \leq_{\bar{I}} a<_{I} b<_{I} c \leq_{\bar{I}} x_{1}$ or

$-x_{0}=a=b<_{\bar{I}} c \leq_{\bar{I}} x_{1}$ or

$-x_{0} \leq_{\bar{I}} a<b=c=x_{1}$.

(A4) Monotonicity For all $f, g \in \mathcal{F}$, if $\bigwedge_{s \in S} \pi_{S}(f) \succsim \pi_{s}(g)$, then $f \succsim g$.

(A5) Uncertainty aversion For all $f, g \in \mathcal{F}$ and $a \in I$, if $f \sim g$, then $m(a ; f, g) \succsim f$.

(A6) Non-degeneracy There exist $f, g \in \mathcal{F}$ such that $f \succ g$.

All these axioms can be captured in a language of first-order logic with:

- two unary predicate symbols $\dot{C}, \dot{I}$ (expressing membership in the subsets $\mathcal{F}_{c}$ and $I$, respectively, of $\mathcal{F}$ ),

- two binary predicate symbols $\dot{亠}_{\text {and }} \dot{<}_{\bar{I}}$,

- $\operatorname{card}(S)$ operator symbols $\dot{\pi}_{s}$,

- and a ternary operation symbol $\dot{m}$.

Henceforth, the language with the predicate symbols $\dot{C}, \dot{I}$, the operator symbols $\dot{\pi}_{s}$ (for each $s \in S$ ), the predicate symbols $\dot{\succsim}$ and $\dot{<}_{\bar{I}}$ the operation symbol $\dot{m}$, and a constant symbol $\dot{f}$ for every element $f \in \mathcal{F}$.

Let $\Gamma$ be the set of all models $\mathfrak{A}=\left\langle\mathcal{F},\left\langle C^{\mathfrak{A}}, I^{\mathfrak{A}}, \succsim^{\mathfrak{A}},<_{\bar{I}}^{\mathfrak{A}},\left\langle\pi_{s}^{\mathfrak{A}}\right\rangle_{s \in S}, m^{\mathfrak{A}}\right\rangle\right\rangle$ of (A1-A6) with domain $\mathcal{F}$ such that $\mathfrak{A}$ canonically interprets

- the constant symbols $\dot{f}$ (i.e. $f^{\mathfrak{A}}=f$ for every $f \in \mathcal{F}$ ),

- the unary predicate symbols $\dot{C}$ and $\dot{I}$ (i.e. $C^{\mathfrak{A}}=\mathcal{F}_{c}$ and $I^{\mathfrak{A}}=I$ ),

- the binary relation symbol $\dot{<}_{\bar{I}}$ (i.e. $\dot{x}^{\mathfrak{A}}<_{I} \mathfrak{A} \dot{y}^{\mathfrak{A}}$ if and only if $x<_{I} y$ for all $x, y \in$ $X)$, 
- the operator symbols $\dot{\pi}_{s}$ (i.e. $\mathfrak{A} \models \dot{\pi}_{s}(\dot{f}) \doteq \dot{g}$ if and only if $f(s)=g$ for all $s \in S$ and $f, g \in \mathcal{F}$ ), and

- the ternary operation symbol $m$ (i.e. $\mathfrak{A} \models \dot{m}(\dot{a} ; \dot{f}, \dot{g}) \doteq \dot{h}$ if and only if $m(a ; f, g)=$ $h$ for all $f, g, h \in \mathcal{F}$ and all $a \in I)$.

Then, elements of $\Gamma$ are in a canonical one-to-one correspondence with variational preference orderings. Hence, variational preference aggregators are in a canonical one-to-one correspondence with maps $G: \operatorname{dom}(G) \rightarrow \Gamma$ where $\operatorname{dom}(G) \subseteq \Gamma^{N}$, such maps $g$ shall also be called model aggregators.

One can now rephrase the variational preference aggregator axioms as model aggregator axioms. Let $\mathcal{T}$ be the Boolean closure of the set of atomic sentences. We shall call $\mathcal{T}$ the set of test sentences.

- universal if and only if $\operatorname{dom}(G)=\Gamma^{N}$ (so that $G: \Gamma^{N} \rightarrow \mathcal{P}$ );

- weakly universal if and only if $\operatorname{dom}(G)$ is a rich aggregator domain. Herein, a set $\mathbb{D}$ is a rich aggregator domain if and only if there exist atomic sentences $\mu, v$ and models $\mathfrak{A}_{1}, \mathfrak{A}_{2}, \mathfrak{A}_{3}$ such that

$-\mathfrak{A}_{1} \models \mu \dot{\wedge} v, \quad \mathfrak{A}_{2}=\mu \dot{\wedge} \dot{\neg} v, \quad \mathfrak{A}_{2} \models \dot{\neg} \mu \dot{\wedge} v$ and

- $\left\{\mathfrak{A}_{1}, \mathfrak{A}_{2}, \mathfrak{A}_{3}\right\}^{N} \subseteq \operatorname{dom}(G)$;

- systematic if and only if for every $\underline{\mathfrak{A}} \in \operatorname{dom}(G)$ and all test sentences $\lambda, \lambda^{\prime}$ satisfying $\left\{i \in N: \mathfrak{A}_{i} \models \lambda\right\}=\left\{i \in N: \mathfrak{A}_{i} \models \lambda^{\prime}\right\}$ one has

$$
G(\underline{\mathfrak{A}}) \models \lambda \Leftrightarrow G(\underline{\mathfrak{A}}) \models \lambda^{\prime} ;
$$

- Paretian if and only if for every $\underline{\mathfrak{A}} \in \operatorname{dom}(G)$ and all test sentences $\lambda$, if $\mathfrak{A}_{I} \models \lambda$ for all $i \in N$, then $G(\mathfrak{A}) \models \lambda$;

- dictatorial if and only if there exists some $i \in N$ (called dictator) such that for every $\underline{\mathfrak{A}} \in \operatorname{dom}(G)$ and all test sentences $\lambda$,

$$
G(\underline{\mathfrak{A}}) \models \lambda \Leftrightarrow \mathfrak{A}_{i} \models \lambda .
$$

A coalition $D$ is said to be decisive with respect to a model aggregator $G$ if and only if there is some $\underline{\mathfrak{A}} \in \operatorname{dom}(G)$ and some test sentence $\lambda$ such that

$$
G(\underline{\mathfrak{A}}) \models \lambda, \quad D=\left\{i \in N: \mathfrak{A}_{i} \models \lambda\right\} .
$$

The set of all decisive coalitions with respect to $G$ is denoted $\mathcal{D}_{G}$.

\section{Appendix C: Proof details}

Let $\Delta$ be the set of all probability measures on $S$. From Theorem 3 of (Maccheroni et al., 2006, Theorem 3), a binary relation $\succsim$ on $\mathcal{F}$ is a variational preference relation if and only if there exists a non-zero linear function $u: X \rightarrow \mathbb{R}$ and a convex lower semi-continuous function $c: \Delta \rightarrow[0,+\infty]$ whose infimum is a real number (rather than $-\infty$ or $+\infty$ ) such that for all $f, g \in \mathcal{F}$, 


$$
f \succsim g \Leftrightarrow \min _{p \in \Delta}\left(\int u \circ f \mathrm{~d} p+c(p)\right) \geq \min _{p \in \Delta}\left(\int u \circ g \mathrm{~d} p+c(p)\right) .
$$

In that case, we say that $\succsim$ has the variational representation $(u, c)$.

Proof of Remark 6 Let $s_{0}, s_{1}$ be two distinct states of the world, and let $u$ be a non-zero linear function. Without loss of generality, assume $u\left(x_{0}\right)<u\left(x_{1}\right)$.

Let $\mathcal{C}_{s_{0}, s_{1}}$ be the set of all convex lower semi-continuous function $c: \Delta \rightarrow[0,+\infty]$ whose infimum is a real number and which satisfy $c(p)=+\infty$ for all $p \in \Delta$ such that $p\{s\}>0$ for some $s \in S \backslash\left\{s_{0}, s_{1}\right\}$. Then, one can define for all $c \in \mathcal{C}_{S_{0}, s_{1}}$ a new function $\widehat{c}:[0,1] \rightarrow \mathbb{R}$ by

$$
\widehat{c}: q \mapsto c\left(q \delta_{s_{0}}+(1-q) \delta_{s_{1}}\right)
$$

(wherein $\delta_{s}$ denotes the Dirac probability measure concentrated on the singleton $\{s\}$ ), and $c$ can be uniquely reconstructed from $\hat{c}$, because $c(p)=+\infty$ holds for all $p \in \Delta$ that are not concentrated on $\left\{s_{0}, s_{1}\right\}$, and any $p \in \Delta$ that is concentrated on $\left\{s_{0}, s_{1}\right\}$ will have the form $c\left(q \delta_{s_{0}}+(1-q) \delta_{s_{1}}\right)$ for some $q \in[0,1]$.

Let $f, g \in \mathcal{F}$ be such that

$$
f\left(s_{0}\right)=x_{0}, \quad f\left(s_{1}\right)=x_{1}, \quad g\left(s_{0}\right)=x_{1}, \quad g\left(s_{1}\right)=x_{0} .
$$

Clearly then one has, for all $c \in \mathcal{C}_{s_{0}, s_{1}}$,

$$
\begin{aligned}
\min _{p \in \Delta}\left(\int u \circ f \mathrm{~d} p+c(p)\right) & =\min _{q \in[0,1]}\left(q u\left(x_{0}\right)+(1-q) u\left(x_{1}\right)+\widehat{c}(q)\right) \\
& =u\left(x_{1}\right)+\min _{q \in[0,1]}\left(q\left(u\left(x_{0}\right)-u\left(x_{1}\right)\right)+\widehat{c}(q)\right) \\
\min _{p \in \Delta}\left(\int u \circ g \mathrm{~d} p+c(p)\right) & =\min _{q \in[0,1]}\left(q u\left(x_{1}\right)+(1-q) u\left(x_{0}\right)+\widehat{c}(q)\right) \\
& =u\left(x_{0}\right)+\min _{q \in[0,1]}\left(q\left(u\left(x_{1}\right)-u\left(x_{0}\right)\right)+\widehat{c}(q)\right)
\end{aligned}
$$

Let us now denote by $c_{1}$ the (by what we remarked above) unique element of $\mathcal{C}_{s_{0}, s_{1}}$ that satisfies $\widehat{c_{1}}(q)=0$ for all $q \in[0,1]$. Then, because $u\left(x_{1}\right)>u\left(x_{0}\right)$ or equivalently $u\left(x_{0}\right)-u\left(x_{1}\right)<0$ and $u\left(x_{1}\right)-u\left(x_{0}\right)>0$, Eqs. (5) and (6) yield

$$
\begin{aligned}
\min _{p \in \Delta}\left(\int u \circ f \mathrm{~d} p+c_{1}(p)\right) & =u\left(x_{1}\right)+\min _{q \in[0,1]}(q\left(u\left(x_{0}\right)-u\left(x_{1}\right)\right)+\underbrace{\widehat{c_{1}}(q)}_{=0}) \\
& =u\left(x_{1}\right)+u\left(x_{0}\right)-u\left(x_{1}\right)=u\left(x_{0}\right) \\
\min _{p \in \Delta}\left(\int u \circ g \mathrm{~d} p+c_{1}(p)\right) & =u\left(x_{0}\right)+\min _{q \in[0,1]}(q\left(u\left(x_{1}\right)-u\left(x_{0}\right)\right)+\underbrace{\widehat{c_{1}}(q)}_{=0}) \\
& =u\left(x_{0}\right)
\end{aligned}
$$


hence, $f \sim_{1} g$ if $\succsim_{1}$ is chosen as the variational preference relation with variational representation $\left(u, c_{1}\right)$.

Next, denote by $c_{2}$ the unique element of $\mathcal{C}_{s_{0}, s_{1}}$ for which $\widehat{c_{2}}(q)=q\left(u\left(x_{1}\right)-u\left(x_{0}\right)\right)$ for all $q \in[0,1]$. Then, again by Eqs. (5) and (6),

$$
\begin{aligned}
\min _{p \in \Delta}\left(\int u \circ f \mathrm{~d} p+c_{2}(p)\right) & =u\left(x_{1}\right)+\min _{q \in[0,1]}\left(q\left(u\left(x_{0}\right)-u\left(x_{1}\right)\right)+\widehat{c_{2}}(q)\right) \\
& =u\left(x_{1}\right) \\
\min _{p \in \Delta}\left(\int u \circ g \mathrm{~d} p+c_{2}(p)\right) & =u\left(x_{0}\right)+\min _{q \in[0,1]}\left(q\left(u\left(x_{1}\right)-u\left(x_{0}\right)\right)+\widehat{c_{2}}(q)\right) \\
& =u\left(x_{0}\right)+\min _{q \in[0,1]} 2 q\left(u\left(x_{1}\right)-u\left(x_{0}\right)\right) \\
& =u\left(x_{0}\right),
\end{aligned}
$$

hence, $f \succ_{2} g$ if $\succsim_{2}$ is chosen as the variational preference relation with variational representation $\left(u, c_{2}\right)$.

Finally, let $c_{3}$ be the unique element of $\mathcal{C}_{s_{0}, s_{1}}$ satisfying $\widehat{c_{3}}(q)=q\left(u\left(x_{0}\right)-u\left(x_{1}\right)\right)$ for every $q \in[0,1]$. Then, Eqs. (5) and (6) imply

$$
\begin{aligned}
\min _{p \in \Delta}\left(\int u \circ f \mathrm{~d} p+c_{3}(p)\right) & =u\left(x_{1}\right)+\min _{q \in[0,1]}\left(q\left(u\left(x_{0}\right)-u\left(x_{1}\right)\right)+\widehat{c_{3}}(q)\right) \\
& =u\left(x_{1}\right)+\min _{q \in[0,1]} 2 q\left(u\left(x_{0}\right)-u\left(x_{1}\right)\right) \\
& =u\left(x_{1}\right)+2\left(u\left(x_{0}\right)-u\left(x_{1}\right)\right) \\
& =2 u\left(x_{0}\right)-u\left(x_{1}\right)<u\left(x_{0}\right) \\
\min _{p \in \Delta}\left(\int u \circ g \mathrm{~d} p+c_{3}(p)\right) & =u\left(x_{0}\right)+\min _{q \in[0,1]}\left(q\left(u\left(x_{1}\right)-u\left(x_{0}\right)\right)+\widehat{c_{3}}(q)\right) \\
& =u\left(x_{0}\right)
\end{aligned}
$$

hence, $g \succ_{3} f$ if $\succsim_{3}$ is chosen as the variational preference relation with variational representation $\left(u, c_{3}\right)$.

All in all, we have found variational preference relations $\succsim_{1}, \succsim_{2}, \succsim_{3}$ with

$$
f \succsim_{1} g \succsim_{1} f, \quad f \succ_{2} g, \quad g \succ_{3} f
$$

If we put $f^{\prime}=g$ and $g^{\prime}=f$, then $f, g, f^{\prime}, g^{\prime}$ and $\succsim_{1}, \succsim_{2}, \succsim_{3}$ satisfy the requirements in the definition of a rich aggregator domain.

It follows that $\mathcal{P}^{N}$ is a rich aggregator domain. Therefore, every universal aggregator is also weakly universal.

Proof of Theorem 7 The reformulation of variational preference aggregation as model aggregation in Appendix 7 permits the application of the impossibility result in Corollary 14 (a generalisation of Arrow's theorem) which in our context says that any weakly universal, systematic, Paretian model aggregator which preserves the (universal) axioms A1-A2, A4-A6 (i.e. the variational preference axioms without continuity) 
is a dictatorship if $N$ is finite. Hence, a fortiori, any strictly variational preference aggregator (which by definition even preserves all axioms A1-A6 on its domain) must be a dictatorship if $N$ is finite.

Proof of Theorem 8 Again, in light of Appendix B, we may use the characterisation of aggregators as restricted ultraproduct constructions (Lemma 16). Note that for fixed $f, g, h$ and $\alpha, \beta, \gamma$, the formula " $\langle\alpha, \gamma\rangle$ is a witness-pair to the continuity of $\succsim$ along $f, g, h$ in $\beta$ " is a universal formula, and all the axioms A1-A2, A4-A6 are also universal formulae. Hence, the axioms A1-A2, A4-A6 as well as the formulae " $\langle\alpha, \gamma\rangle$ is a witness-pair to the continuity of $\succsim$ along $f, g, h$ in $\beta$ " (for all fixed $f, g, h, \alpha, \beta, \gamma$ ) are preserved by restricted ultraproducts. Therefore, restricted ultraproduct constructions on rich domains are model aggregators which not only are weakly universal, systematic and Paretian and preserve axioms A1-A2, A4-A6, but they also aggregate equicontinuous profiles into continuous profiles. Hence, any restricted ultraproduct construction on a rich domain with only equicontinuous profiles constitutes a weakly universal, systematic and Paretian strictly variational preference aggregator. However, on an infinite set $N$ there are non-principal ultrafilters and, thus, non-dictatorial aggregators derived from restricted ultraproduct constructions.

Proof of Lemma 10 This is a direct consequence of Lemma 12, itself a slight generalisation of the main lemma in Lauwers and Van Liedekerke (1995, Lemma 2).

\section{Appendix D: variational preference aggregation with restricted domain through majority voting}

We have seen that in general, universal systematic Paretian aggregation of convex risk measures is impossible. If one drops universality, then rational aggregation of risk measures is still possible, viz. through majority voting about risk measures, but at the expense of considerable restrictions on the variational preference profiles.

In analysing majority decisions about convex risk measures, one can build on the work of Dietrich and List (2010) who have developed a theory of majority voting in the very general framework of judgment aggregation, including a generalisation of May (1952) theorem (which uniquely characterises majority voting by means of certain axioms such as anonymity and acceptance/rejection neutrality). To do so, one has to embed the aggregation problem for risk measures into the framework of judgment aggregation.

Consider the axiom system $\Sigma$ consisting of the following formulae:

- The axioms (A1-A6) as reformulated in Appendix B.

- The formulae $\dot{\neg} \dot{f} \doteq \dot{g}$ for all $f, g \in \mathcal{F}$ such that $f \neq g$.

- All formulae of the form $\dot{\pi}_{s}(\dot{f}) \doteq \dot{x}$ for all $s \in S, f \in \mathcal{F}$ and $x \in \mathcal{F}_{c}$ satisfying $\pi_{s}(f)=x$.

- All formulae of the form $\dot{C} \dot{x}$ for all $x \in \mathcal{F}_{c}$, and all formulae of the form $\dot{\neg} \dot{C} \dot{f}$ for all $f \in \mathcal{F} \backslash \mathcal{F}_{c}$.

- All formulae of the form $\dot{I} \dot{a}$ for all $a \in I$, and all formulae of the form $\dot{\neg} \dot{I} \dot{f}$ for all $f \in \mathcal{F} \backslash I$. 
- All formulae of the form $\dot{m}(\dot{a} ; \dot{f}, \dot{g})=\dot{h}$ for all $a \in I$ and $f, g, h \in \mathcal{F}$ satisfying $m(a ; f, g)=h$.

For any set of $\mathcal{L}$-formulae $A$, completeness and consistency will be understood to mean $\Sigma$-completeness and $\Sigma$-consistency

Let

$$
\Xi=\{\dot{f} \dot{\succsim} \dot{g}, \dot{\neg} \dot{f} \dot{\succsim} \dot{g}: f, g \in \mathcal{F}\}
$$

This is an agenda in the terminology of judgment aggregation, i.e. a set of propositionnegation pairs. In the following, for any $p \in \Xi$, we mean by $\neg p$ the other element of the proposition-negation pair in $\Xi$ to which $p$ belongs, so that $\neg \neg p=p$ for any $p \in \Xi$.

A fully rational judgment set is a complete and consistent subset of $\Xi$; note that by the choice of $\Sigma$, any fully rational judgment set uniquely determines a preference relation $\succsim$ on $\mathcal{F}$ that satisfies axioms (A1-A6). The set of fully rational judgment sets will be denoted by $D$.

Let $N$ be finite. A profile is an $N$-tuple $\underline{A}=\left(A_{i}\right)_{i \in N}$ of fully rational judgment sets. For each profile $\underline{A}$ and any $p \in \Xi$, we define the coalition supporting $p$ under profile $\underline{A}$ by

$$
\underline{A}(p)=\left\{i \in N: p \in A_{i}\right\} .
$$

The aggregation rule of majority voting is then defined as the map

$$
F: D^{N} \rightarrow 2^{\Xi}, \quad \underline{A} \mapsto\{p \in \Xi: \operatorname{card}(\underline{A}(p))>\operatorname{card}(\underline{A}(\neg p))\} .
$$

At least whenever $\operatorname{card}(N)$ is odd, the aggregate judgment set $F(\underline{A})$ will be complete for every $\underline{A} \in D^{N}$. The question is whether $F(\underline{A})$ will be consistent as well; if it is, it is a fully rational judgment set and thus by our observation made above, uniquely determines a preference relation $\succsim$ on $\mathcal{F}$ that satisfies axioms (A1-A6), hence a convex risk measure.

A sufficient condition for the consistency of $F(\underline{A})$ for certain $\underline{A} \in D^{N}$ has been discovered by Dietrich and List (2010) and is known as the value-restriction property. A profile $\underline{A} \in D^{N}$ value-restricted is for every non-singleton, minimal inconsistent subset $Y \subseteq \Xi$ there exists a two-element subset $Z \subseteq Y$ such that $Z \nsubseteq A_{i}$ for all $i \in N$. If there is an ordering on the agenda with respect to which every $A_{i}$ (for $i \in N$ ) is (locally) single-plateaued or single-canyoned, then the profile is value-restricted and hence $F(\underline{A})$ is consistent (and complete anyway).

However, in the case of variational preference profiles, the conditions of singlecanyonedness or single-plateauedness - let alone the value-restriction property-do not appear to be natural conditions. This gives additional weight to the impossibility result for finite electorates in the present paper. 


\section{References}

Arrow, K.J.: Social Choice and Individual Values. Cowles Commission Monographs, vol. 12, 2nd edn. Wiley, New York (1963)

Bell, J.L., Slomson, A.B.: Models and Ultraproducts. An Introduction. North-Holland, Amsterdam (1969)

Bossert, W., Weymark, J.A.: Social Choice: Recent Developments. Cahier de recherche \# 2006-01, Université de Montréal (2006)

Campbell, D.E.: Intergenerational social choice without the Pareto principle. J. Econ. Theory 50(2), 414423 (1990)

Cerreia-Vioglio, S., Ghirardato, P., Maccheroni, F., Marinacci, M., Siniscalchi, M.: Rational preferences under ambiguity. Econ. Theory 48(2-3), 341-375 (2011)

Chateauneuf, A., Eichberger, J., Grant, S.: A simple axiomatization and constructive representation proof for Choquet expected utility. Econ. Theory 22(4), 907-915 (2003)

Chateauneuf, A., Eichberger, J., Grant, S.: Choice under uncertainty with the best and worst in mind: neo-additive capacities. J. Econ. Theory 137(1), 538-567 (2007)

de Castro, L.I., Chateauneuf, A.: Ambiguity aversion and trade. Econ. Theory 48(2-3), 243-273 (2011)

Detlefsen, K., Scandolo, G.: Conditional and dynamic convex risk measures. Finance Stoch. 9(4), 539-561 (2005)

Dietrich, F., List, C.: Arrow's theorem in judgment aggregation. Soc. Choice Welf. 29(1), 19-33 (2007)

Dietrich, F., List, C.: Judgment aggregation without full rationality. Soc. Choice Welf. 31(1), 15-39 (2008)

Dietrich, F., List, C.: The Aggregation of Propositional Attitudes: Towards a General Theory. Oxford Studies in Epistemology, vol. 3, pp. 215-234. Oxford University Press, Oxford (2010)

Dietrich, F., List, C.: Majority voting on restricted domains. J. Econ. Theory 145(2), 512-543 (2010)

Dietrich, F., Mongin, Ph.: The Premiss-Based Approach to Logical Aggregation. Les Cahiers de Recherche 886, HEC Paris (2007)

Dokow, E., Holzman, R.: Aggregation of binary evaluations. J. Econ. Theory 145(2), 495-511 (2010)

Dominiak, A., Lefort, JPh: Agreement theorem for neo-additive beliefs. Econ. Theory. (2012). doi:10.1007/ s00199-011-0678-7

Ellsberg, D.: Risk, ambiguity, and the Savage axioms. Q. J. Econ. 75(4), 643-669 (1961)

Ellsberg, D., Machina, M.J., Ritzberger, K., Yannelis, N.C.: Introduction to the symposium issue [Symposium on the 50th Anniversary of the Ellsberg Paradox]. Econ. Theory 48(2-3), 219-227 (2011)

Fishburn, P.C.: Arrow's impossibility theorem: concise proof and infinite voters. J. Econ. Theory 2(1), 103-106 (1970)

Föllmer, H., Schied, A.: Convex measures of risk and trading constraints. Finance Stoch. 6(4), 429-447 (2002)

Föllmer, H., Schied, A.: Stochastic Finance. An Introduction in Discrete Time. de Gruyter Studies in Mathematics, vol. 27, 2nd edn. de Gruyter, Berlin (2004)

Gajdos, T., Tallon, J.M., Vergnaud, J.C.: Representation and aggregation of preferences under uncertainty. J. Econ. Theory 141(1), 68-99 (2008)

Gilboa, I., Samet, D., Schmeidler, D.: Utilitarian aggregation of beliefs and tastes. J. Polit. Econ. 112(4), 932-938 (2004)

Gilboa, I., Schmeidler, D.: Maxmin expected utility with non-unique prior. J. Math. Econ. 18(2), 141-153 (1989)

Hansen, L.P., Sargent, T.J.: Robust control and model uncertainty. Am. Econ. Rev. 91(2), 60-66 (2001)

Harsanyi, J.C.: Cardinal welfare, individualistic ethics, and interpersonal comparisons of utility. J. Polit. Econ. 63(4), 309-321 (1955)

Herzberg, F.S.: A representative individual from Arrovian aggregation of parametric individual utilities. J. Math. Econ. 46(6), 1115-1124 (2010)

Herzberg, F.S., Eckert, D.: Impossibility results for infinite-electorate abstract aggregation rules. J. Philos. Log. 41(1), 273-286 (2012a)

Herzberg, F.S., Eckert, D.: The model-theoretic approach to aggregation: impossibility results for finite and infinite electorates. Math. Soc. Sci. 64(1), 41-47 (2012b)

Herzberg, F.S., Lauwers, L., Van Liedekerke, L., Fianu, E.S.: Addendum to L. Lauwers and L. Van Liedekerke, "Ultraproducts and aggregation". J. Math. Econ. 46(2), 277-278 (2010)

Hodges, W.: Building Models by Games. London Mathematical Society Student Texts, vol. 2. Cambridge University Press, Cambridge (1985) 
Hylland, A., Zeckhauser, R.J.: The impossibility of Bayesian group decision making with separate aggregation of beliefs and values. Econometrica 47(6), 1321-1336 (1979)

Kalai, E., Muller, E., Satterthwaite, M.A.: Social welfare functions when preferences are convex, strictly monotonic, and continuous. Public Choice 34(1), 87-97 (1979)

Keisler, H.J.: Model Theory for Infinitary Logic. Logic with Countable Conjunctions and Finite Quantifiers. Studies in Logic and the Foundations of Mathematics, vol. 62. North-Holland, Amsterdam (1971)

Kirman, A.P., Sondermann, D.: Arrow's theorem, many agents, and invisible dictators. J. Econ. Theory 5(2), 267-277 (1972)

Knight, F.H.: Risk, uncertainty, and profit. Hart, Schaffner and Marx, Boston (1921)

Lauwers, L., Van Liedekerke, L.: Ultraproducts and aggregation. J. Math. Econ. 24(3), 217-237 (1995)

Le Breton, M.: Essais sur les fondements de l'analyse économique de l'inégalité. Thèse pour le Doctorat d'État en Sciences Économiques, Université de Rennes 1, 1986

Le Breton, M., Weymark, J.A.: Social choice with analytic preferences. Soc. Choice Welf. 19, 637-657 (2002)

Le Breton, M., Weymark, J.A.: Arrovian social choice theory on economic domains. In: Arrow, K.J., Sen, A., Suzumura, K. (eds.) Handbook of Social Choice and Welfare, vol. 2, page Chapter 17. North-Holland, Amsterdam (2010)

List, C., Pettit, P.: Aggregating sets of judgments: an impossibility result. Econ. Philos. 18, 89-110 (2002)

List, C., Puppe, C.: Judgment aggregation: a survey. In: Anand, P., Pattanaik, P.K., Puppe, C. (eds.) The Handbook of Rational and Social Choice: An Overview of New Foundations and Applications, pp. 457-482. Oxford University Press, Oxford (2009)

Loś, J.: Quelques remarques, théorèmes et problèmes sur les classes définissables d'algèbres. In: Skolem, Th., Hasenjaeger, G., Kreisel, G., Robinson, A., Wang, H., Henkin, L., Łoś, J. (eds.) Mathematical Interpretation of Formal Systems. Studies in Logic and the Foundations of Mathematics, vol. 16, pp. 98-113. North-Holland, Amsterdam (1955)

Maccheroni, F., Marinacci, M., Rustichini, A.: Ambiguity aversion, robustness, and the variational representation of preferences. Econometrica 74(6), 1447-1498 (2006)

Maccheroni, F., Marinacci, M., Rustichini, A.: Dynamic variational preferences. J. Econ. Theory 128(1), 4-44 (2006)

Maskin, E.: Social welfare functions for economics. Cambridge University, Mimeo (1976)

Maskin, E.: Fonctions de préférence collective définies sur des domaines de préférence individuelle soumis a des contraintes. Cahiers du Séminaire d'Économétrie 20, 153-182 (1979)

May, K.O.: A set of independent necessary and sufficient conditions for simple majority decision. Econometrica 20(4), 680-684 (1952)

McConway, K.J.: Marginalization and linear opinion pools. J. Am. Stat. Assoc. 76(374), 410-414 (1981)

Mongin, Ph: Consistent Bayesian aggregation. J. Econ. Theory 66(2), 313-351 (1995)

Mongin, Ph: The paradox of the Bayesian experts and state-dependent utility theory. J. Math. Econ. 29(3), 331-361 (1998)

Moulin, H.: On strategy-proofness and single peakedness. Public Choice 35(4), 437-455 (1980)

Nau, R.: Risk, ambiguity, and state-preference theory. Econ. Theory 48(2-3), 437-467 (2011)

Nehring, K., Puppe, C.: The structure of strategy-proof social choice. Part I: general characterization and possibility results on median spaces. J. Econ. Theory 135(1), 269-305 (2007)

Neuefeind, W., Trockel, W.: Continuous linear representability of binary relations. Econ. Theory 6, 351-356 (1995)

Riedel, F.: Dynamic coherent risk measures. Stoch. Process. Appl. 112(2), 185-200 (2004)

Seidenfeld, T., Kadane, J.B., Schervish, M.J.: On the shared preferences of two Bayesian decision makers. J. Philos. 86(5), 225-244 (1989)

von Neumann, J., Morgenstern, O.: Theory of Games and Economic Behavior. Princeton University Press, Princeton (1944)

Waszkiewicz, J., Wȩglorz, B.: On products of structures for generalized logics. Studia Log 25, 7-15 (1969)

Yu, N.N.: A one-shot proof of Arrow's impossibility theorem. Econ. Theory 50(2), 523-525 (2012) 